\title{
Audience size and likelihood and intensity of response during a humorous movie
}

\author{
SHELDON G. LEVY \\ Wayne State University, Detroit, Michigan 48202
}

and

WILLIAM F. FENLEY, JR.

Houston, Texas

\begin{abstract}
The relationship between audience size and audience response was examined through unobtrusive observations of individuals attending the movie "M.A.S.H." Between three and six observers attended 15 different showings of the movie in the same theater. The distribution of the observers throughout the theater reflected the distribution of the audience, and each observer noted the response of specified individuals to a set of 25 scenes that had been selected during pretesting with actual audiences. The evidence strongly supported the hypothesis that likelihood of response would increase with increasing audience size. Based on the evidence, a social facilitation rather than a conformity interpretation seems to be supported.
\end{abstract}

Historical and theoretical observations together with empirical studies have examined the influence of group reaction on individual response. Freud (1960), LeBon (1960), Rude (1959), and Smelser (1962) stressed that a prerequisite for mass behavior is some unifying force or connection. Once this connection is postulated, the influence of the group as well as the spread of emotion throughout the group is a logical consequence. Further, emotional contagion has been hypothesized to be a function of group size (McDougall, 1920).

The effects of an audience's presence have been experimentally studied. Zajonc (1966) reviewed these studies and concluded that the presence of others increases general arousal and facilitates dominant responses while hindering learning. Based on work which began with G. H. Allport, Zajonc concluded that an individual's actions are enhanced by others doing the same thing, that is, social facilitation occurs in coacting groups.

In addition, empirical studies (e.g., Asch, 1955; Rosenthal, 1963) have established that individual response is altered by group pressure. Rosenthal found that subjects exposed to a "commonly held" opinion about a photograph prior to their own evaluation tended to reflect that opinion in their evaluation. Asch's research was, of course, limited to small groups in the laboratory. For those judgments that were examined, conformity effects reached an asymptote as

This research was initially conducted as part of an advanced undergraduate seminar on the psychology of mass behavior at Rice University. We thank David Grant, Jade Schiffman, Mike Young, and Paul Goldstein for their assistance on the project. Requests for reprints should be sent to Sheldon G. Levy, Department of Psychology, Wayne State University, Detroit, Michigan 48202. the number of influencers reached as few as three members. However, Milgram (reported in Milgram and Toch, 1969) conducted a field study and found that the effect on passersby increased through the maximum size of 15 precipitators. Finally, Argyle (1958), using data from other sources, reported statistics on Billy Graham meetings in Europe in 1954 and 1955. These data indicate that the percentage of the audience that made a decision tended to increase with larger audiences. However, the percentages also increased through time from 1954 to 1955. Therefore, other factors may be involved rather than audience size.

The present study was concerned with the effects of audience size upon group response. It was felt that nonreactive observations of a naturally occurring group would make the greatest contribution to this area of research (Webb, Campbell, Schwartz, \& Sechrest, 1966) for several reasons. First, such observations allow examination of groups larger than those in the laboratory. Second, effects in a real social situation could be examined. Third, the dependent variable would be a voluntary response which the subject anitcipated providing in that situation.

The hypothesis tested was that there would be an increase in the percentage of persons responding to a given stimulus and an increase in the mean intensity of the response as group size increased.

\section{METHOD}

\section{Preliminary Study}

The movie "M.A.S.H." was selected and attended several times by the entire group of six observers. The reactions of subjects were noted, with particular attention paid to the following: (1) ability to observe subjects in a darkened theater, (2) location and number of subjects most easily observed, 
(3) types of responses, and (4) types of scenes eliciting visible response.

Based on this study, a representative set of 25 scenes was chosen that adhered to the following criteria: (1) Most of the scenes had moderate response levels to allow for meaningful measurement of audiences varying in receptivity. (2) Scenes were selected to assure sampling of the entire movie. (3) Sufficient time was allowed between scenes to buffer the chosen scenes from the residual effects of the other selected scenes. That is, an effort was made to select scenes sufficiently removed in time so that the responses should be independent of each other.

Observers indicated the difficulty of attempting to record all types of responses. The most easily recordable behavior was visible laughter, which became the operational definition of audience response.

No difficulty was reported in noting responses if the subjects were limited to a few persons seated in the immediate vicinity of the observer. Further, observers were able to remain unobtrusive, since subjects either did not notice the note taking or thought the notes were being taken on the movie itself, not the audience.

\section{Experimental Study}

Each observer recorded the response of three subjects to predetermined scenes on a 3-point scale: 0 , no laughter; 1 , moderate laughter; 2, sustained laughter.

Although the distinction between moderate and sustained laughter is more difficult than that between laughter vs. no laughter, added reliability is generally obtained by increasing the scale points. For example, the more ambiguous task of measuring a subject's opinions on an attitude scale is frequently aided by allowing the subject to indicate amount of agreement or disagreement rather than merely agree or disagree. (See discussion under Reliability.)

Observers were placed so that sampling was proportional to the physical distribution of the audience. Observations were restricted to three subjects seated either immediately in front of the observer or across an aisle if the observer occupied a seat at the end of a row.

Responses were recorded for each subject for 25 predetermined scenes. Fifteen different audiences were studied, ranging in size from 125 to 976 in a theater in Houston, Texas, with a capacity of 976 .

\section{RESULTS}

The audience response is presented in Table 1 for each size.

The correlation between audience size and mean response was .97. (Between size and percentage of " 0 " responses, it was -.95 ; between audience size and percentage of " 2 " responses, it was +.97 .) The results were significant beyond the .001 level.

The relationship between audience size and response is further shown through the correlations with each scene separately. Of the 25 Pearson rs, 21 were significant beyond the .05 level and 16 of those beyond the .001 level.

\section{Reliability}

The reliability of judges' ratings of subject laughter was evaluated using two different procedures. First, reliability was examined for two judges observing different subjects at the same showing of the film. This
Table 1

Audience Response Percentages with Increasing Audience Size

\begin{tabular}{cccccc}
\hline & & & \multicolumn{3}{c}{$\begin{array}{c}\text { Amount of Laughter } \\
\text { (Percent Response) }\end{array}$} \\
\cline { 5 - 6 } $\begin{array}{c}\text { Audience } \\
\text { Size }\end{array}$ & $\begin{array}{c}\text { Ob- } \\
\text { servers }\end{array}$ & $\begin{array}{c}\text { Sub- } \\
\text { jects }\end{array}$ & 0 & 1 & 2 \\
\hline 125 & 3 & 9 & 44.0 & 29.8 & 26.2 \\
198 & 4 & 12 & 47.4 & 25.6 & 27.0 \\
273 & 4 & 12 & 40.6 & 28.3 & 31.3 \\
286 & 6 & 18 & 37.6 & 28.4 & 34.0 \\
394 & 4 & 12 & 35.3 & 34.4 & 30.3 \\
504 & 4 & 12 & 30.0 & 32.7 & 37.3 \\
543 & 6 & 18 & 29.8 & 34.2 & 36.0 \\
575 & 4 & 12 & 31.6 & 27.8 & 40.6 \\
666 & 5 & 15 & 30.7 & 26.0 & 43.3 \\
740 & 5 & 15 & 29.9 & 22.9 & 47.2 \\
813 & 4 & 12 & 24.4 & 29.3 & 46.3 \\
845 & 4 & 12 & 23.3 & 29.3 & 47.4 \\
945 & 4 & 12 & 25.3 & 26.0 & 48.7 \\
976 & 6 & 18 & 22.0 & 31.3 & 46.7 \\
976 & 6 & 18 & 20.7 & 29.9 & 49.4 \\
\hline
\end{tabular}

is obviously a most stringent test of interjudge agreement, since it requires both reliability in judges' ratings and homogeneity of subject response across the audience. Across audience sizes the average gamma coefficient of +.70 indicated very substantial agreement.

Reliability of the judges' ratings was further tested on a second film ("Harold and Maude") using the following procedure. One judge was informed about the hypothesis of the study, while a second judge remained naive. In addition, the ears of the naive judge were covered to mask the sound of the audience. (It was not possible to completely mask the sound since the judge would have then been unable to respond adequately to the movie cues.) The two judges then each observed three subjects within the theater. Two of the subjects were observed by both judges and one subject was unique to each judge. The procedure was repeated for two different showings of the film. For subjects observed by both judges, gamma $=+.99$. For subjects observed separately at the same showing, gamma for the judges was +.72 , thus confirming almost precisely the first reliability analysis. ${ }^{1}$

It should also be noted that the above measures were based on comparisons of two judges' ratings. Since the actual data are based on the combined observations of as many as six judges at each showing of the movie, reliability of the data used in this investigation should be at least as high as that presented above.

\section{Conformity vs. Social Facilitation}

The study's results can best be interpreted as indicating the effects of social facilitation, thus supporting McDougall's (1920) hypothesis as well as supporting the experimental research reported by Zajonc (1966).

However, it is also reasonable to ask if there was a conformity effect. That is, were responses of part of the audience dependent on the responses of others? There are two possible ways to examine this problem. 
The first is to consider that, for any particular scene, the response of some members (the followers) occurred after the response of others (the leaders). It is not possible to investigate this aspect of the conformity problem without precise measuring instruments. However, the effect of conformity can operate in a second way, to increase the responsiveness in subsequent scenes. In the second circumstance one should expect two results. First, responsiveness should increase from one scene to the next. Second, one might expect greater conformity pressures the larger the audience.

The Pearson $r$ of scene number with mean scene response for all sizes taken together was $.43(\mathrm{p}<.01$ for 25 scenes). Further, correlations of scene number with mean scene response within each size indicated that all $r$ were positive $\left(p=1 / 2^{14}=.00006, n=14\right.$ since the two audiences with $n=976$ were combined). Five of the correlations were significant beyond .01 .

Therefore, responsiveness increased throughout the film. However, it is quite possible that this was merely due to an increase in the intrinsic humor of the film. Since this determination is difficult to make independently of human judgment, an argument can be made that if conformity, rather than intrinsic humor, is the influence, the rate of increase in response intensity should be greater for the larger than for the smaller audiences.

The evidence does not support this contention. Correlations across scenes did not increase in strength from the smallest to the largest audience size. Further, the slopes of the responses remained similar across scenes from one audience size to the next. (The smallest Pearson $r$ between any two curves was +.97.) These results are presented in Figure 1. The lowest curve represents audience sizes $125-286$, the middle represents 394-576, and the highest curve, 666-976.

Each point on the diagram is based on the average intensity of laughter per scene for the set of audience sizes, that is,

$$
\sum_{\mathrm{j}=\mathrm{S}_{\mathrm{r}}}^{\mathrm{S}_{\mathrm{t}=\mathrm{a}} \mathrm{a}+4} \overline{\mathrm{X}}_{\mathrm{i}, \mathrm{j}} /[(\mathrm{t}-\mathrm{r}+1) \cdot 5],
$$

where $\mathrm{j}$ is the audience size, $\mathrm{r}$ is the rank of the audience size (from smallest to largest), $i$ is the scene number, a equals $1,6,11,16$, or 21 , and $\bar{X}_{i, j}$ is the mean response for scene $i$ and the audience size $j$. Thus the first point on the lowest curve is based on

$$
\sum_{\mathrm{j}=\mathrm{S}_{1}}^{\mathrm{S}_{4}} \underset{\mathrm{i}=1}{5} \overline{\mathrm{X}}_{\mathrm{i}, \mathrm{j}} / 4 \cdot 5
$$

where $S_{1}=125, S_{2}=198, S_{3}=273$, and $S_{4}=286$.

Figure 1 also indicates that the assumption that extended group pressure should lead to more homo-

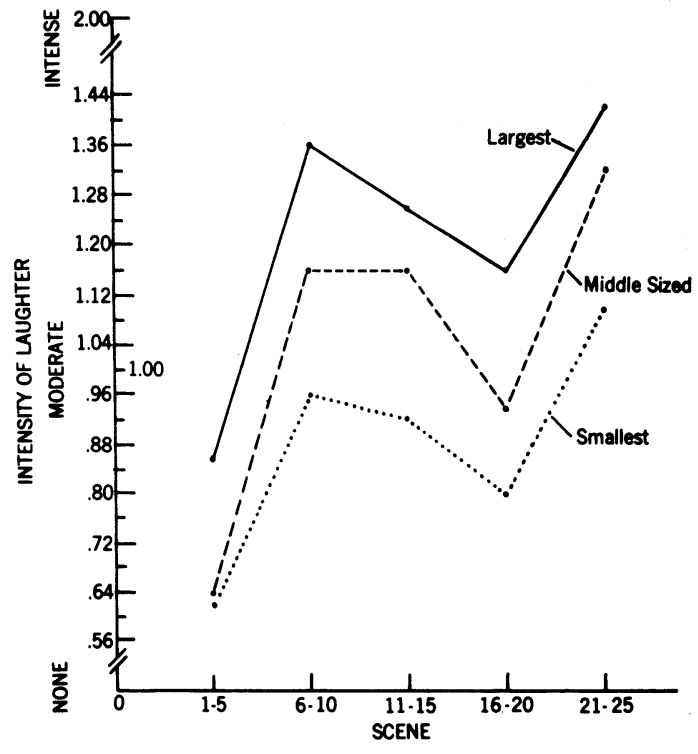

Figure 1. Relationship between intensity of laughter and scene for various audience sizes.

geneous responses with larger audience size is not supported. According to this view, not only should an increase in laughter from $\bar{X}_{i}$ to $\bar{X}_{i+1}$ be followed by larger increases from $\overline{\mathrm{X}}_{\mathrm{i}+1}$ to $\overline{\mathrm{X}}_{\mathrm{i}+2}$ for bigger audiences but also decreases from $\bar{X}_{i}$ to $\bar{X}_{i+1}$ should be followed by larger decreases from $\bar{X}_{i+1}$ to $\bar{X}_{i+2}$.

\section{DISCUSSION}

Earlier writings on the effects of crowds on the individual have been based on subjective impressions of natural events that were not always observed directly. Later experimental work controlled variables within, of necessity, artificial environments. This study examined the effects of audience size upon individual responsiveness through precise but nonreactive observations of naturally occurring groups (Webb et al., 1966).

The reason for the increased probability and intensity of the responses is difficult to ascertain in natural settings. Two explanations may be conformity and social facilitation. If conformity is operating, one would expect that: (1) responsiveness should increase throughout the film if the conformity effect is cumulative (although the intrinsic humor of the film could obviously affect these results, as could fatigue); (2) the larger the audience, the greater should be the rate of the increases and decreases in laughter.

Although responsiveness did increase throughout the film, the rates of increase did not agree with the predictions from the conformity hypothesis. Consequently, a tentative conclusion would be that the findings in this study were due to social facilitation rather than conformity.

However, the judgment of conformity effects cannot absolutely be answered by this research. One reason is that the smallest audience size was 125 and whatever conformity effects existed might have leveled off by the time that size was reached. Second, all scenes were not observed. Thus, it is possible that there were short-term effects that could not be ascertained. Finally, interfering factors such as intrinsic humor of the scenes and of individual fatigue could not be measured. However, the almost precisely constant level of increases from one curve to the next with increasing audience size does indicate that effects 
are attributable to audience size. Intrinsic humor variations appear to be of consequence only within a particular size. These conclusions are clearly indicated in Figure 1. There was no interaction between scene number (which included the intrinsic humor) and audience size.

It is hoped that this research will contribute to the design of precise but nonreactive procedures for measuring the effects of the presence and behavior of crowds upon the individual.

\section{REFERENCES}

Argyle, M. Religious behavior. London: Routledge and Kegan Paul. 1958.

Asch, S. E. Opinions and social pressure. Scientific American, 1955, 193 (5), 31-35.

FREUD, S. Group psychology and the analysis of the ego. New York: Bantam, 1960.

LeBon, G. The crowd, a study of the popular mind. New York: Viking, 1960. (First published in French, 1895).

McDougall, W. The group mind (collective psychology). New York: Putnam, 1920.

Milgram, S., \& Toch, H. Collective behavior: Crowds and social movements. In G. Lindzey \& E. Aronson (Eds.), The handbook of social psychology (Vol. 4, 2nd ed.). Reading, Mass: Adddison-Wesley, 1969.
Rosenthal, R. The experimenter's hypothesis as unintended determinants of experimental results. American Scientist, 1963, 51. 268-283.

Rude, G. The crowd in the French revolution. Oxford: Oxford University Press, 1959.

SMelser, N. J. Theory of collective behavior. New York: Free Press of Glencoe, 1962.

Webb. E. J., Campbell, D. J., Schwartz, R. D., \& Sechrest, L. Unobtrusive measures: Nonreactive research in the social sciences. Chicago: Rand McNally, 1966.

Zajonc. R. G. Social psychology: An experimental approach. Belmont, Calif: Wadsworth, 1966.

\section{NOTE}

1. Although gamma only measures agreement in direction of change, it does indicate that reported differences in audience reaction as a function of audience size are not attributable to the idiosyncracies of the judge. Although level of laughter observed may differ from one judge to the next, changes in levels are reliably predicted. Examination of the specific judgments of the raters in the reliability study showed precise agreement as to the subject's score in 65 of 79 observations.

(Received for publication February 23, 1979.) 